\title{
TRANSMISSION LOSS ESTIMATION OF UNDERWATER SOUND BASED ON THE NOISE INTENSITY EMMITED BY MV. PENGAYOMAN IV IN TANJUNG INTAN CRUISE LINE, CILACAP
}

\author{
Lesa Triwahyanti ${ }^{1}$, Ajeng Sekar Cyndana ${ }^{1}$, Yessy Hurly Sefnianti ${ }^{1}$, Ratna Juita \\ Sari ${ }^{1}$, Amron Amron ${ }^{1 *}$ \\ ${ }^{1}$ Marine Science Department, Jenderal Soedirman University, Kampus Unsoed \\ Karangwangkal, Purwokerto 53122 Indonesia
}

\begin{abstract}
The water is an imperfect acoustic medium, acoustic energy can lost due to absorption and scattering thus resulting in transmission loss. The value of transmission loss in water is an important thing to know, for example in the application of active acoustic. This study aim is to determine the value of transmission loss in Tanjung Intan's cruise line, Cilacap. The acquisition of vessel's sound data was obtained by hydrophone and vessel data (distance) by CCTV. Both sound data and vessel data were connected to personal computer to record the audio and visual signal synchronously for three consecutive days. Results showed that vessel characteristics such as intensity, frequency and interval duration have a value of $43.35(\mathrm{~dB}), 6613.54(\mathrm{~Hz})$ and 216.15 (ms) respectively. While the value of water absorption coefficient has a range of $500-900 \mathrm{~dB} / \mathrm{km}$, the estimated transmission loss value was between $5-11 \mathrm{~dB} / \mathrm{km}$.
\end{abstract}

Corresponding author : twoone brond@scientist..com 


\section{INTRODUCTION}

Indonesia is the biggest archipelago in the world which is largely surrounded by oceans. This allows the development of technology to optimize the potential of Indonesia in the field of shipping, fisheries, military, and another matters related to Indonesia's potential in maritime field. One of them is the development of underwater communication. The ability to communicate effectively in underwater is important for marine research, oceanographers, commercial marine operators, oil industry, and defense. Underwater communication method can use acoustic or electromagnetic method. Both of these methods propagate waves through the medium and will be diffused, reflected and absorbed by the water. However, the acoustic method has less absorption than electromagnetic so can reach longer distances in the water medium [1].

The water medium is an imperfect acoustic medium. When the sound spreads through the water, acoustic energy can be lost by absorption and scattering [1]. Urick [2] adds that acoustic wave propagation will through the process of loss of intensity caused by geometrical spreading loss and absorption loss. This is known as propagation loss (transmission loss) which is one of the sonar parameters that express a quantitative value of some phenomena related to the propagation of acoustic waves at sea.

According to Duncan and Parsons [3], calculating transmission loss is complicated by its dependence on many different factors, including the horizontal separation of the source and receiver, their positions in the water column, the temperature and salinity profiles of the water column, the water depth, the slope and roughness of the seabed, the geology of the seabed, and the roughness of the sea surface. Whereas according to Burnett [4], transmission loss is influenced by environmental and non-environmental factors. Environmental factors that affect such as sea conditions, surface temperatures, depth profiles of temperature and also daily and seasonal geographic variations. While nonenvironmental factors such as transmitter and receiver depth, transmission range and frequency. Hence the stochastic process characterizing transmission loss is implicitly indexed by the corresponding transmission and receiver depths and transmission range.

The value of transmission loss in water is important to know, for example in active acoustic application. According to study conducted by Lermusiaux et al. [5], the prediction of transmission loss values can be used to determine the geoacoustic model of the type and thickness of the sediment. In addition, it can also be used to find out the bathymetry and condition of the water column. Transmission loss can also be used to know the dynamics of the tides in the water column. Meanwhile, according to Jones et al. [6], acoustic transmission modeling can be used to view the presence of water column features.

\section{MATERIALS AND METHODS}

The research was conducted on 22-25 January 2018 for $3 \times 24$ hours. Sampling was conducted on MV. Perkasa 7 that leaning on the dock of Pandu Station, Cilacap Central Java (7044'30"' S 108059'49" T). MV.Pengayoman IV which is the object in this study sailed in the Tanjung Intan cruise line, Cilacap. The method used observation method by recording the sound intensity of MV.Pengayoman IV used hydrophone and ship activity used CCTV. 


\subsection{Data Acquisition}

Data acquisition has been done for $3 \times 24$ hours. Underwater sounds was recorded used a Hydrophone Sea Phone SQ26-H1 was at depth of 1-2 meters and ship activity as support data was recorded used CCTV (Fig. 1).

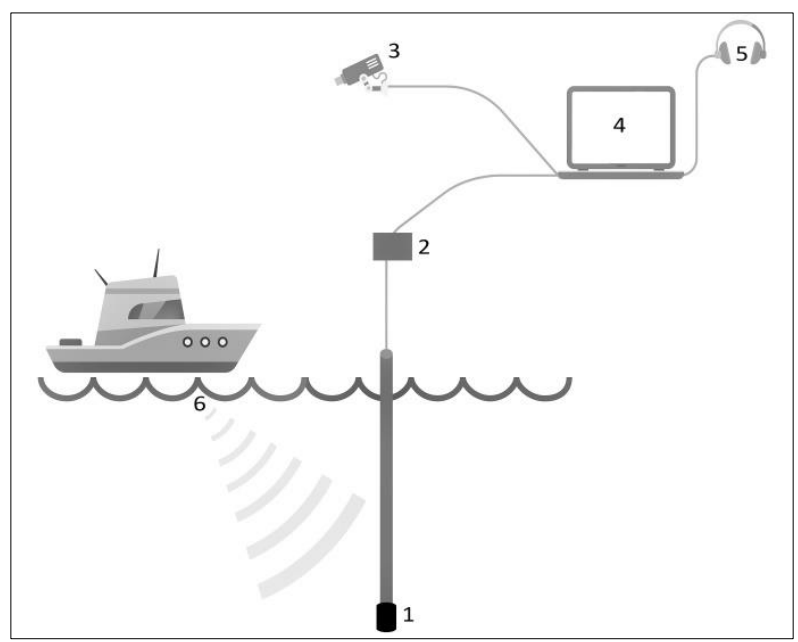

Fig 1. Instrumentation preparation design

\subsection{Data Processing}

\subsubsection{Measurement of Vessel Distance}

The distance of the vessel to the hydrophone was measured by projecting the vessel's position on CCTV into the Google Earth Software. The observed vessels were sought the position and distance to the hydrophone so can be seen the difference of resulted sound intensity. To simplify the positioning of the vessel, used fixed objects around the cruise line to be projected as buoy, wijayapura ferry port and cement mining (Fig. 2).

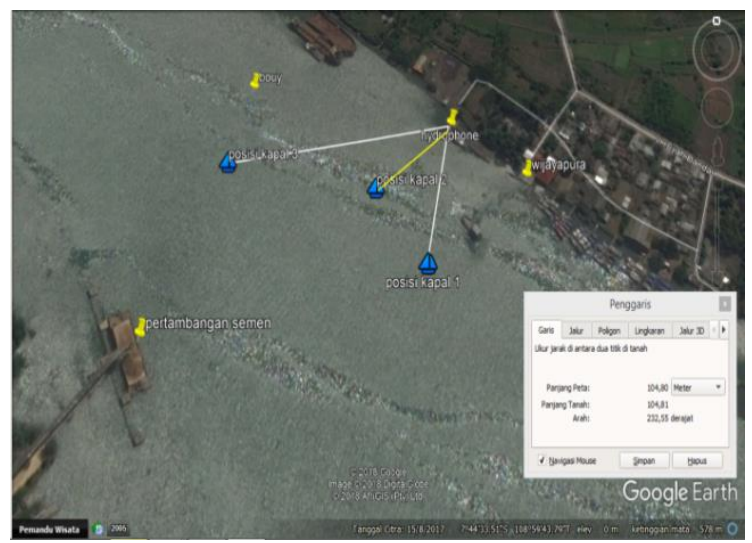

Fig 2. Measuring Vessel distance from hydrophone 


\subsubsection{Data Processing of Vessel Noise Characteristics}

Passive acoustic systems will generate a lot of raw data. The recorded data contains the sound required in the study (sound of the vessel) and not required (natural sounds such as wind, rain, waves or mining activity around the study site). Therefore, prior to data analysis, sound processing was required to separate the required sound and not required by Wavelab 6.1 software. Stages performed were Envelope, Spectrum, and Sound Characteristic determination.

\subsubsection{Transmission Losses Value Calculation}

Absorption loss and geometric spreading loss on the acoustic wave propagation will give the transmission loss value according to the equation [2] which can be expressed in equation (1):

$$
\mathrm{TL}=20 \log \mathrm{R}+\alpha \mathrm{R}
$$

Where the $\mathrm{R}$ factor (Distance) is entered because the $\alpha$ is usual expressed in $\mathrm{dB} / \mathrm{km}$ (or $\mathrm{dB} /$ kyard). Since the absorption coefficient value $(\alpha)$ has not been obtained, the TL value is calculated using ordinary least square estimation (equation.2):

$$
\mathrm{Y}=\mathrm{a}-\mathrm{bx}
$$

Where $\mathbf{Y}$ is a representation of the intensity at $\mathbf{X}$ distance, $\mathbf{a}$ is a representation of the initial intensity and $\mathbf{b x}$ is the value of transmission loss.

\section{RESULTS AND DISCUSSION}

\subsection{Vessel Noise Characteristic}

MV. Pengayoman IV is one type of ship crossing ferry or also called Ro-Ro ships. Roll-on/roll-off (RORO or Ro-Ro) ships are vessels designed to carry wheeled cargo, such as cars, trucks, and motorcycle that are driven on and off the ship on their. MV. Pengayoman IV has a specification length of $21 \mathrm{~m}$, speed $4 \mathrm{~km} /$ hour and capacity 127 GT. Dussardier [7] stated that the use of Horse Power 3-3.5 times the Gross Tonnage of the vessel. The vessel produces noise characteristics as in table 1 . This result was obtained by recording distance between 35-80 meters from hydrophone.

Table 1. Average result of noise characteristic of MV.Pengayoman IV .

\begin{tabular}{lllll}
\hline Ship Name & $\begin{array}{l}\text { Ship } \\
\text { Size }\end{array}$ & $\begin{array}{l}\text { Intensit } \\
\text { y (-dB) }\end{array}$ & $\begin{array}{l}\text { Frequenc } \\
\text { y (Hz) }\end{array}$ & $\begin{array}{l}\text { Interval } \\
\text { Duratio } \\
\text { n (ms) }\end{array}$ \\
\hline $\begin{array}{l}\text { Kapal } \\
\begin{array}{l}\text { Pengayoman } \\
\text { IV }\end{array}\end{array}$ & $\begin{array}{l}127 \\
\text { GT }\end{array}$ & 43,35 & 6613,54 & 216.15 \\
\hline
\end{tabular}




\subsection{Coeficient Absorption}

When acoustic waves propagate in water, some of the sound energy will be absorbed by water and converted into heat [1]. This absorption occurs through two main mechanisms including the viscosity of the seawater itself and the molecular relaxation. According to Urick [2], molecular relaxation is a major cause of absorption loss at acoustic frequencies below $100 \mathrm{kHz}$. At high frequencies (more than $500 \mathrm{kHz}$ ), acoustic pressure changes too quickly to avoid molecular relaxation, and absorption of acoustic energy is absent. Here is the graph of coefficient of absorption based on frequency:

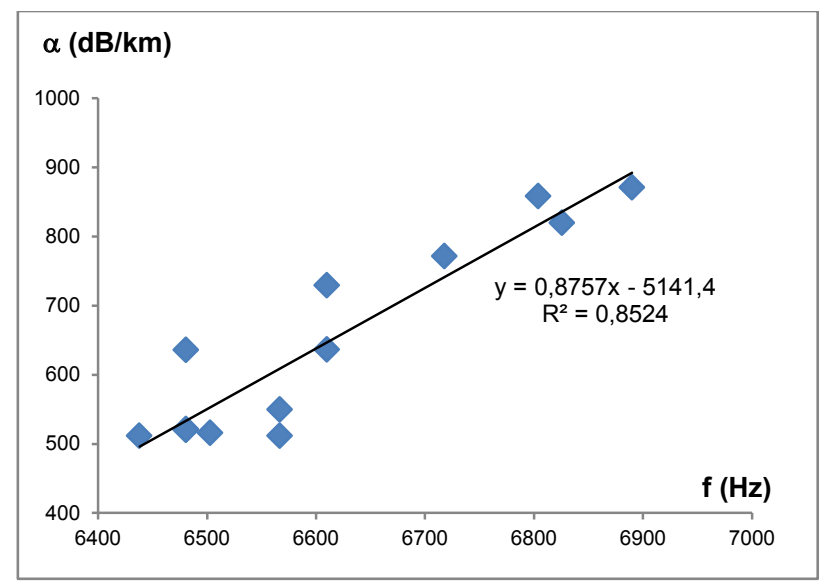

Fig 3. Graph of frequency to absorption coefficient value

The amount of energy absorbed $(\alpha)$ will depend on various factors. In other words $\alpha$ is a function of the sound frequency, pressure, temperature, and density of the water [8]. The value of the absorption coefficient will increase as the frequency. The frequency range obtained was $6400-6800 \mathrm{~Hz}$ and the absorption coefficient was $500-900 \mathrm{~dB} / \mathrm{km}$.

\subsection{Transmission Loss Estimation}

Transmission loss is generally influenced by non-environmental factors such as transmitter and receiver depth, transmission range and frequency. Therefore, the stochastic process that controls transmission loss is implicitly indexed by the transmission distance, the depth of the receiver and the transmission range [4]. The estimated transmision loss value that was affected by the transmission distance of 35-80 m ranges from 4.80 to 11.02 dB/km (Fig. 4). 


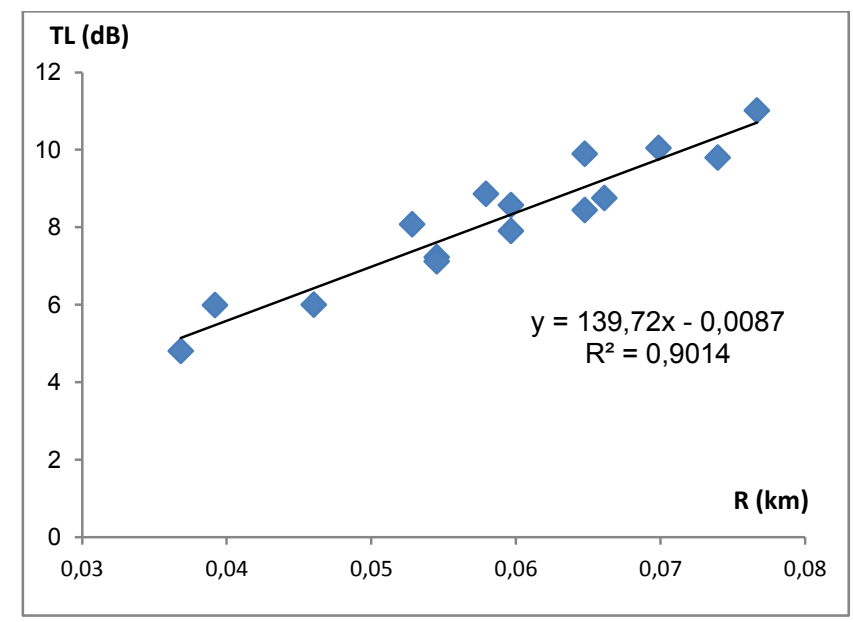

Fig 4. The value of transmission loss based on the distance traveled by sound waves

Various activities on the surface of the waters by various commercial vessels produce a significant contribution to underwater noise [9]. The main source of vessel noise is when the vessel speed is low because the components of the diesel generator (SSDG) donate all the noise from the vessel. When the vessel speed is high, the noise source comes from the dominant drive of the vessel. Basically every vessel has a vibration source. The biggest vibrations occur in the engine room. This is because the engine room has main engine that works as the main driver of the vessel. Vibration on the vessel produces varying noise levels according to the distance from the vibration source and the attenuation that inhibits the propagation of noise from the vibration source [10].

Based on the research that has been done, MV. Pengayoman IV resulted noise characteristic of intensity $43.35(\mathrm{~dB})$, frequency $(6613.54 \mathrm{~Hz})$ and interval duration 216.15 miliseconds. While previous research conducted by Lesage and Barrete [11] on ferry ship with specification length 80 meter, speed $28 \mathrm{~km}$ / hour, size $2173 \mathrm{GT}$ and the power 2000 $\mathrm{HP}$ was produced noise intensity characteristic equal to 40-75 (-dB) and frequency 175$6000 \mathrm{~Hz}$. According to Ross [17] anthropogenic sound as in the shipping activity has a frequency reaching $10 \mathrm{KHz}$. Changes of noise characteristics can be used as a reference in the transmission process in the water.

According to Preisig [12], acoustic propagation through seawater is characterized by a significant frequency of absorption and relatively slow speed propagation. The value of the absorption coefficient will increase along with the increase in frequency (Fig 3). As pressure waves propagate through water, the transfer of thermal energy associated with chemical relaxation processes drives the absorption of sound by seawater salts [13].

The frequency is proportional to the absorption coefficient. Conceptually, higher frequencies involve faster particle velocities and thus higher friction losses. In seawater the relaxation of certain compounds can cause additional losses owing. For each compound, there is a critical 'relaxation' frequency below which the losses occur. The induced pressure reduction of molecules to ions which takes a certain time to complete is called relaxation. At high frequencies, there is no energy that absorbed because sound pressure cycles too quick for the reduction to occur and thus by this process. The magnesium sulphate relaxation dominates the absorption in the frequency range $2-500 \mathrm{kHz}$, and at lower frequencies there is a further loss associated with boric acid. The frequency is the main determinant of the absorption, but $\alpha$ also depends on the water temperature and salinity[1]. 
The limiting factor which determines the highest useful frequency for the detection of targets at a given range is rapid increase of absorption with frequency. The received signal is below the noise level and cannot be detected because the absorption loss is so great. While the absorption in freshwater is relatively small at low frequencies, since the molecular relaxation loss is absent [1]. Coefficient absorption is one of the transmission loss parameters.

The accumulated decrease in acoustic intensity as an acoustic pressure wave propagates outwards from a source can be defined as transmission loss. The effects of a variety of propagation phenomena in the sea is summarizes by transmission loss. Adding the effects of geometrical spreading, absorption and scattering, then transmission loss can estimated [14].

Chitre [15] assumes that the energy intensity at each wave surface point will be reduced as the square of the distance traveled by the waves and transmission loss increases according to the square of the distance. Based on the study conducted by Duncan et a.1 [16] in $10 \mathrm{~km}$ distance, range of transmission loss between $75-90(\mathrm{~dB} / \mathrm{km})$. While in $80 \mathrm{~km}$ distance, range of transmission loss will increase between 110-120 (dB/km). In Fig. 4, the increased transmission loss was proportional to the increase of distance. The statement is justified by [4], transmission loss is implicitly caused by the transmission distance, the depth of the receiver and the transmission range.

\section{CONCLUSION}

The characteristics of ship noise consist of intensity, frequency, and duration of intervals. Changes in noise characteristics can be used as a guide in determining the value of transmission loss. The absorption coefficient is one of the parameters that can affect the transimission loss. The value of the absorption coefficient increases with increasing frequency, and the value of transmission loss increases with distance.

Authors gratefully acknowledge to Pelindo III, Pandu Station and the Capt of MV. Perkasa 07 for the permits, places and facilities provided in

\section{REFERENCE}

1 E.J. Simmonds, D.N. Maclennan. Fisheries Acoustics, $2^{n^{d}}$ edition. (Blackwell Science, Oxford,UK, 2005)

2 R.J. Urick. Principles of Underwater Sound. 3rd Edition. (McGraw-Hill, New York, 1983)

3 A.J. Duncan, M.C.G. Parsons. Proceedings of Acoustic 2011 (Gold Coast, Aus. Acous.Soc, 2011)

4 T.D. Burnett. The Caracterization of Variability in Transmission Loss in The Ocean. (Naval Postgraduate School, California, 1971)

5 P.F.J. Lermusiaux, X. Jinshan, F.C. Chi, J. Sen, Y.C. Linus, J.Y. Yiing. IEEE, 35, 895916 (2011) 
6 A.D. Jones, Z. Alex, V.G. Michael. Proceedings of Acoustics 2013 (Victor Harbor, Australian Acoustical Society, 2013)

7 A. Dussardier. Consideration on Dissels. Fishing Boat of the World II. (Jan-olof Traung London, Fishing News (Book) Ltd, 1960)

8 J. Widodo. Oseana. 14, 81-92 (1989)

9 M F. McKenna, D. Ross, S.M . Wiggins, J.A. Hildebrand. America. J. Acous. Soc. 131 92-103 (2012)

10 C.S. Clay, H. Medwin. Accoustical Oceanoghraphy: Principles and Aplications. (John Wiley and Sons, New York, 1998)

11 V. Lesage, C. Barrete. Mar. Mammal Sci, 15, 65-84 (1999)

12 J. Preisig. Mobile Computing and Communications Review. 11, 2-10 (2006)

13 I.J. Doonan, R.F. Coombs, S. McClatchie. J.Mar.Sci. 60, 1047-1055 (2003)

14 M.C. Domingo, R. Prior. Computer Communication. 31, 1227-1238 (2008)

15 M. Chitre. Underwater Acuostic Communications in Warm Shallow Waters Channels. Ph.D. Thesis. (Electrical and Computer Engineering. National University of Singapore, Singapore, 2006)

16 A.J. Duncan, A. N. Gavrilov, R. D. McCauley, and M. Parnum America. J. Acous. Soc.134, 201-216 (2013)

17 D. Ross. Mechanics of Underwater Noise. (Pergamon, New York, 1976) 